\title{
UNCERTAINTY MODELING VIA FREQUENCY DOMAIN MODEL VALIDATION
}

\author{
Martin R. Waszak, ${ }^{*}$ NASA Langley Research Center, Hampton, Virginia \\ Dominick Andrisani II, ${ }^{\dagger}$ Purdue University, West Lafayette, Indiana
}

\begin{abstract}
The majority of literature on robust control assumes that a design model is available and that the uncertainty model bounds the actual variations about the nominal model. However, methods for generating accurate design models have not received as much attention in the literature. The influence of the level of accuracy of the uncertainty model on closed loop performance has received even less attention. The research reported herein is an initial step in applying and extending the concept of model validation to the problem of obtaining practical uncertainty models for robust control analysis and design applications. An extension of model validation called 'sequential validation' is presented and applied to a simple spring-mass-damper system to establish the feasibility of the approach and demonstrate the benefits of the new developments.
\end{abstract}

\section{Introduction}

Robust control theory guarantees that a feedback control system can be designed that will maintain desired levels of stability and performance subject to modeling errors and uncertainties. ${ }^{[1,2]}$ There is, however, an underlying assumption that the uncertainty model used in the design effectively characterizes the differences between the responses of the true system and the nominal design model. This means that the family of responses associated with the design model contain the responses of the true system.

It is impossible to conclusively prove that this assumption is satisfied for any real system. However, if there is sufficient knowledge of the response characteristics of the true system then a model can be generated that, subject to the available knowledge, characterizes the possible range of responses that can be produced by the system. The goal is to systematically generate such a model with the added

\footnotetext{
* Senior Research Engineer. Senior Member AIAA.

$\dagger$ Associate Professor, School of Aeronautics and Astronautics. Senior Member AIAA.

Copyright (C) 1999 by the American Institute of Aeronautics and Astronautics, Inc. No copyright is asserted in the United States under Title 17, U.S. Code. The U.S. Government has a royalty-free license to exercise all rights under the copyright claimed herein for Governmental purposes. All other rights are reserved by the copyright owner.
}

property that it be accomplished with minimal conservatism. That is, the model should characterize responses of the real system that are possible but should not characterize responses that are not possible. Of course, it is impossible to conclusively prove this as well. However, if the available knowledge is comprehensive enough it is possible to achieve this goal within some qualitative level of confidence.

Model Validation

A concept called "model validation" has recently been developed to attack this problem. ${ }^{[3-8]}$ The idea behind model validation is that given input and output data for a system that is otherwise unknown, a model can be generated that driven by the same input (and possibly some additional inputs) can exactly reproduce the output of the true system. The frequency domain version of this statement is depicted in block diagram form in Figure 1.

The input to the true system $\mathbf{u}(\mathbf{s})$ produces the output of the true system $\mathbf{y}(\mathbf{s})$. The same input drives the model along with an external disturbance $\mathbf{w}(\mathbf{s})$. Another external input $\mathbf{v}(\mathbf{s})$ is added to the response of the model to produce the validation output $\tilde{\mathbf{y}}(\mathbf{s})$. The external inputs are included because the true system output includes artifacts of the method(s) by which it is obtained including external disturbances, estimation errors, and quantization effects. The external inputs provide a mechanism to account for these effects. Validation is achieved by choosing the model and the external inputs $\mathbf{w}(\mathbf{s})$ and $\mathbf{v}(\mathbf{s})$ so that the difference between the validation output and the true system output $\mathbf{e ( s )}$ is identically zero for the available input/output data. (The Laplace variable $\mathbf{s}$ will be omitted henceforth for ease of discussion.)

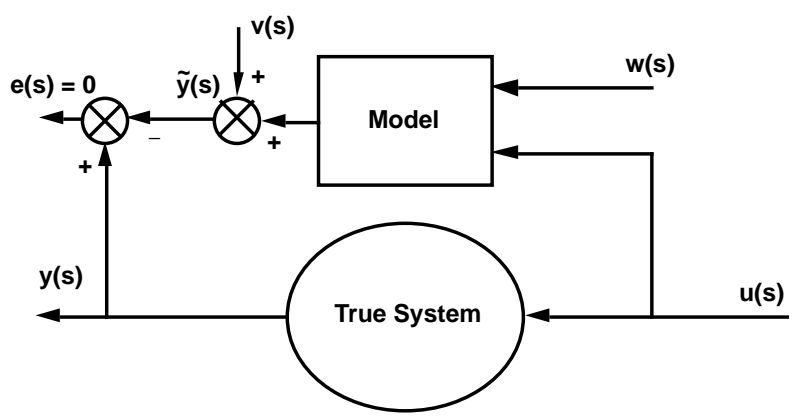

Figure 1 - Model validation block diagram. 
The goal is to choose the validating model and external inputs in such a way that the resulting model can be used for robust control design. There are infinitely many ways to choose a validating model and so the next step is to develop a means for generating a validating model that can be used in robust control design and demonstrate reduced levels of conservatism.

\section{Robust Control Models}

Robust control design requires a linear design model that characterizes parameter variations, unmodeled dynamics, and/or other uncertainties relative to a nominal model. The common format for describing such a model in block diagram form is shown in Figure $2 .{ }^{[1,2]}$ The nominal model is represented by the $(2,2)$ element of the transfer matrix $\mathbf{P}$ while the other elements represent various aspects of the uncertainty. The matrices $\Omega$ and $\Delta_{\mathbf{B}}$ characterize the structure of the uncertainty in the model; $\Omega$ is a real diagonal positive definite weighting matrix and $\Delta_{\mathbf{B}}$ is a block diagonal matrix with unity norm (a so-called unit ball). The elements of $\Delta_{\mathbf{B}}$ are only specified as to whether they are real or complex valued, satisfy a particular block diagonal structure, and satisfy the norm bound condition.

For a given set of inputs $\mathbf{u}, \mathbf{v}$, and $\mathbf{w}$, the output of the uncertain model $\tilde{\mathbf{y}}$ represents a family of outputs determined by all possible choices for $\Delta_{\mathbf{B}}$ that satisfy the prescribed structure. One measure of conservatism for the uncertain model described above is the size of the external inputs $\mathbf{v}, \mathbf{w}$ and the norm of uncertainty weighting matrix $\Omega$. A reasonable goal would be to generate a validating model that minimizes these quantities.

The model validation problem can be broken down into several sub-problems. First input/output data from the true system must be obtained. It may come from experiment, actual operation, simulation, or computed directly from a high-fidelity model that is used to represent the true system. The second sub-problem is choosing a structure for the model. Robust control design relies on a linear model called a linear fractional transformation (LFT) ${ }^{[1,2]}$ and is represented by the block diagram in Figure 2. The equation for the LFT in Figure 2 is

$$
\begin{aligned}
\tilde{\mathbf{y}}= & {\left[\mathbf{P}_{\mathbf{2 3}}+\mathbf{P}_{\mathbf{2 1}} \Delta_{\mathbf{B}} \Omega\left[\mathbf{I}-\mathbf{P}_{11} \Delta_{\mathbf{B}} \Omega\right]^{-\mathbf{1}} \mathbf{P}_{\mathbf{1 3}}\right]\left\{\begin{array}{l}
\mathbf{v} \\
\mathbf{w}
\end{array}\right\} } \\
& +\left[\mathbf{P}_{\mathbf{2 2}}+\mathbf{P}_{\mathbf{2 1}} \Delta_{\mathbf{B}} \Omega\left[\mathbf{I}-\mathbf{P}_{11} \Delta_{\mathbf{B}} \Omega\right]^{-\mathbf{1}} \mathbf{P}_{12}\right] \mathbf{u}
\end{aligned}
$$

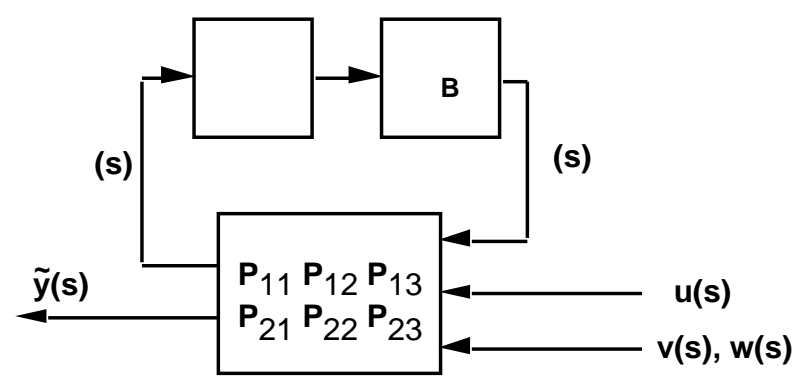

Figure 2 - Robust control model block diagram.

Choosing the model structure involves selecting the $\mathbf{P}_{\mathbf{j}}$, the block structure of $\Omega$ and $\Delta_{\mathbf{B}}$, and the external inputs $\mathbf{V}$ and $\mathbf{w}$. The $\mathbf{P}_{\mathbf{j}}$ arise from the form of the nominal model and the representation of uncertainty (e.g., real or complex valued parameter uncertainty, additive uncertainty, input or output multiplicative uncertainty in unstructured or structured forms). Subsequently the form of $\Omega$ and $\Delta_{\mathbf{B}}$ are chosen consistent with the uncertainty representations. The third sub-problem is setting up an optimization problem that solves for the smallest external inputs and uncertainty weights that, subject to the choice of model structure, makes the validation error $\mathbf{e}$ (from Figure 1) zero.

\section{Extending the Model Validation Method}

The current formulation of the model validation problem optimizes on a single scalar uncertainty weight with a bound constraint on the magnitude of the external inputs. ${ }^{[3]}$ There may be other formulations that may be better able to incorporate the available knowledge. An alternate, more general, approach is proposed and developed in this paper.

The choice for the structure of the uncertainty plays a major role in the relative conservatism of the resulting validating model. How does one choose the uncertainty structure upon which the method operates to get the "best" model? It may be possible to parameterize the potential uncertainty model structures and utilize optimization to select the best structure. An approach to address this issue by means of sequential uncertainty modeling is proposed and developed here as well.

A brief review of the key technical aspects of the model validation method will be presented first. This will be followed by a discussion of the extensions that have been made to the method as part of the current research. This is followed by simple examples that demonstrate the application of the model validation method to generate an uncertainty model for a simple dynamic system. 
The perspective on model validation and uncertainty modeling addressed here is a direct extension of the work of Lim and Giesy. ${ }^{[3]}$ The model validation problem statement and solution is theirs and the current work builds on that foundation. It is summarized and interpreted here to provide a basis for the extensions described subsequently.

\section{Model Validation Problem ${ }^{[3,4]}$}

Assume that input/output data for a physical dynamic system is available and that a robust control design model for the system is desired. Assume further that a linear model, specified uncertainty structure, and a specified level of external disturbance is available for the same system. The model elements can be described as a linear fractional transformation (LFT) as shown in Figure 2. The input data for the physical system can be used as input to the LFT to produce another set of output data. Model validation is achieved by selecting certain elements of the LFT and its external inputs in such as way as to make the difference between the outputs of the physical system and LFT model exactly zero at each frequency. Figure 1 depicts the model validation problem in block diagram form. In equation form model validation is simply

$$
\mathbf{0} \equiv \mathbf{e}=\mathbf{y}-\tilde{\mathbf{y}}=\mathbf{e}_{\mathbf{y}}^{0}-\mathbf{P}_{23}\left\{\begin{array}{l}
v \\
w
\end{array}\right\}-\mathbf{P}_{21} \xi
$$

where

$$
e_{y}^{0}=y-P_{22} u
$$

Recall that the nominal linear model $\mathbf{P}_{\mathbf{2 2}}$ and the input data $\mathbf{u}$ are given. Therefore the quantities $\mathbf{P}_{\mathbf{2 3}}\left\{\begin{array}{ll}\mathbf{v}^{\mathbf{T}} & \mathbf{w}^{\mathbf{T}}\end{array}\right\}^{\mathbf{T}}$ * and $\mathbf{P}_{\mathbf{2 1}} \xi$ can be used to characterize the differences (errors, uncertainties, etc.) between the true system outputs and the model outputs. Validation is achieved by solving Eqn. (2) for these quantities subject to the specified structure of the model.

The model structure is described by the LFT in Eqn. (1). The specified uncertainty structure is represented by $\mathbf{P}_{\mathbf{i}}, \mathbf{i}, \mathbf{j} \neq \mathbf{2}$ and $\Delta_{\mathbf{B}}$. The remaining free variables are $\mathbf{v}$ and $\mathbf{w}$ and the uncertainty weights in $\Omega$. Note that $\Omega$ has a diagonal structure dictated by the structure of $\Delta_{\mathbf{B}}$ which is block diagonal. There is one free weight $\omega_{\mathbf{i}}$ associated with each block of $\Delta_{\mathbf{B}}$ as shown in Eqn. (3) where the $\mathbf{I}_{\mathbf{n}}, \mathbf{n}=\mathbf{1 , 2}, \ldots, \tau$ are identity matrices with dimension equal to the

\footnotetext{
* Note that the superscript $\mathbf{T}$ is used herein to denote complex conjugate transpose.
}

dimension of the $\Delta_{\mathbf{B}_{\mathbf{i}}}, \mathbf{i}=\mathbf{1 , 2}, \ldots, \tau$ and $\tau$ is the number of uncertainty blocks.

$$
\begin{aligned}
\Omega & =\left[\begin{array}{llll}
\omega_{\mathbf{1}} \mathbf{I}_{\mathbf{1}} & & & \\
& \omega_{\mathbf{2}} \mathbf{I}_{\mathbf{2}} & & \\
& & \ddots & \\
& & & \omega_{\tau} \mathbf{I}_{\tau}
\end{array}\right] \\
\Delta_{\mathbf{B}} & =\left[\begin{array}{llll}
\Delta_{\mathbf{B}_{\mathbf{1}}} & & & \\
& \Delta_{\mathbf{B}_{2}} & & \\
& & \ddots & \\
& & & \Delta_{\mathbf{B}_{\tau}}
\end{array}\right]
\end{aligned}
$$

Each $\Delta_{\mathbf{B}_{\mathbf{i}}}$ are unit norm matrices with specified structure (diagonal or full, real or complex) but whose elements are otherwise arbitrary. Therefore the free variables that can be selected to achieve model validation are $\mathbf{v}, \mathbf{w}, \omega_{\mathbf{i}}$, and $\Delta_{\mathbf{B}_{\mathbf{i}}}, \mathbf{i}=\mathbf{1}, \mathbf{2}, \ldots, \tau$.

The current model validation problem formulation combines $\mathbf{v}$ and $\mathbf{w}$ into a single vector. In addition, it is assumed that these signals satisfy the Euclidean norm $^{\dagger}$ bound constraint

$$
\left|\begin{array}{l}
\mathbf{v} \\
\mathbf{w}
\end{array}\right| \leq 1
$$

This comes from the notion that the external inputs are associated with some random phenomena that are unknown except that they are stable and bounded.

\section{Model Validation Solution ${ }^{[3]}$}

The solution of the model validation problem described above is accomplished using a linear vector space approach. The model validation equation, Eqn. (2), can be rewritten

$$
\mathbf{e}_{\mathbf{y}}^{\mathbf{0}}=\mathbf{M}\left\{\begin{array}{l}
\xi \\
\mathbf{v} \\
\mathbf{w}
\end{array}\right\}
$$

where $\mathbf{M}=\left[\begin{array}{ll}\mathbf{P}_{\mathbf{2 1}} & \mathbf{P}_{\mathbf{2 3}}\end{array}\right]$ and $\mathbf{e}_{\mathbf{y}}^{\mathbf{0}}, \mathbf{P}_{\mathbf{2 1}}$, and $\mathbf{P}_{\mathbf{2 3}}$ are known. A necessary condition for Eqn. (5) to be satisfied is that $\mathbf{e}_{\mathbf{y}}^{\mathbf{0}}$ lie in the subspace spanned by the columns of $\mathbf{M}$, that is

$$
\mathbf{e}_{\mathbf{y}}^{\mathbf{0}} \in \operatorname{Im}(M)
$$

The general solution of Eqn. (5) can be written as

\footnotetext{
$\dagger$ All norms referred to herein are Euclidean norms.
} 


$$
\left\{\begin{array}{c}
\xi \\
\mathbf{v} \\
\mathbf{w}
\end{array}\right\}=\mathbf{M}^{+} \mathbf{e}_{\mathbf{y}}^{\mathbf{0}}+\mathbf{N}_{\mathbf{M}} \boldsymbol{\theta}
$$

where

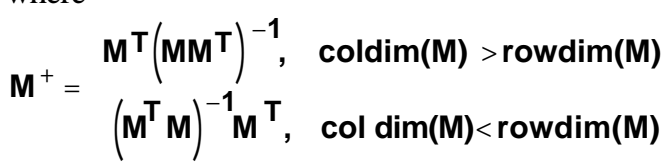

and where the columns of $\mathbf{N}_{\mathbf{M}}$ form a basis for the null space of $\mathbf{M}$. Note that the vector $\boldsymbol{\theta}$ is arbitrary because when it is premultiplied by $\mathbf{N}_{\mathbf{M}}$ the product lies in the null space of $\mathbf{M}$ which when premultiplied by $\mathbf{M}$, as in Eqn. (5), is identically zero. However, $\theta$ does contribute to the norm of $\left[\xi^{\mathbf{T}} \mathbf{v}^{\mathbf{T}} \mathbf{w}^{\mathbf{T}}\right]^{\mathbf{T}}$. Also recall that the vector formed by $\mathbf{v}$ and $\mathbf{w}$ has a norm bound constraint. Therefore, $\boldsymbol{\theta}$ is arbitrary only to the degree to which the norm bound is satisfied.

In order to assure that this constraint is satisfied consider a partition of Eqn. (7)

$$
\left\{\begin{array}{l}
\mathbf{v} \\
\mathbf{w}
\end{array}\right\}=\left(\mathbf{M}^{+}\right)_{\mathbf{v w}} \mathbf{e}_{\mathbf{y}}^{\mathbf{0}}+\left(\mathbf{N}_{\mathbf{M}}\right)_{\mathbf{v w}} \boldsymbol{\theta}
$$

where the subscript $\mathbf{v w}$ represents taking the rows of the respective matrix associated with $\mathbf{v}$ and $\mathbf{w}$. Now $\left(\mathbf{N}_{\mathbf{M}}\right)_{\mathbf{v}}$ can be represented in terms of its singular value decomposition by

$$
\left(\mathrm{N}_{\mathrm{M}}\right)_{\mathrm{vw}}=\left[\begin{array}{ll}
\mathrm{U}_{1} & \mathrm{U}_{2}
\end{array}\right]\left[\begin{array}{cc}
\mathrm{S}_{1} & 0 \\
0 & 0
\end{array}\right]\left[\begin{array}{c}
\mathrm{V}_{1}^{\top} \\
\mathrm{V}_{2}^{\mathrm{T}}
\end{array}\right]
$$

The columns of the matrices $\mathbf{V}_{\mathbf{1}}$ and $\mathbf{V}_{\mathbf{2}}$ span the image and null spaces of $\left(\mathbf{N}_{\mathbf{M}}\right)_{\mathbf{v w}}$, respectively. Therefore, $\theta$ can be written as the sum of two vectors, one in the image of $\left(\mathbf{N}_{\mathbf{M}}\right)_{\mathbf{v w}}$ and one in the null space of $\left(\mathbf{N}_{\mathbf{M}}\right)_{\mathbf{v w}}$,

$$
\theta=\mathbf{V}_{\mathbf{1}} \gamma+\mathbf{V}_{\mathbf{2}} \psi
$$

Note that because $\psi$ multiplies $\mathbf{V}_{\mathbf{2}}$ which spans the null space of $\left(\mathbf{N}_{\mathbf{M}}\right)_{\mathbf{v w}}$ it does not contribute to the norm of $\left\{\begin{array}{c}\mathbf{v} \\ \mathbf{w}\end{array}\right\}$ and is therefore completely arbitrary. However, $\gamma$ does contribute to the norm of $\left\{\begin{array}{l}\mathbf{v} \\ \mathbf{w}\end{array}\right\}$ and is therefore constrained. To determine the constraint on $\gamma$ substitute the expression for $\theta$ from Eqn. (10) and the expression for $\left(\mathbf{N}_{\mathbf{M}}\right)_{\mathbf{v w}}$ from Eqn. (9) into
Eqn. (8). The properties of the singular value decomposition of $\left(\mathbf{N}_{\mathbf{M}}\right)_{\mathbf{v w}}$ allow considerable simplification and the elimination of $\psi$ from the expression.

$$
\left\{\begin{array}{l}
\mathbf{v} \\
\mathbf{w}
\end{array}\right\}=\left(\mathbf{M}^{+}\right)_{\mathbf{v w}} \mathbf{e}_{\mathbf{y}}^{\mathbf{0}}+\mathbf{U}_{\mathbf{1}} \mathbf{S}_{\mathbf{1}} \gamma
$$

Recall the norm bound on $\mathbf{v}$ and $\mathbf{w}$ from Eqn. (4) and note that the matrix $\left[\begin{array}{ll}\mathbf{U}_{\mathbf{1}} & \mathbf{U}_{\mathbf{2}}\end{array}\right]$ is unitary so that the constraint in Eqn. (4) can be written

$$
1 \geq\left|\begin{array}{l}
v \\
w
\end{array}\right|^{2} \equiv\left\|\left[\begin{array}{l}
U_{1}^{T} \\
U_{2}^{T}
\end{array}\right]\left\{\begin{array}{l}
v \\
w
\end{array}\right\}\right\|^{2} .
$$

Premultiplying Eqn. (11) by $\left[\begin{array}{ll}\mathbf{U}_{\mathbf{1}} & \mathbf{U}_{\mathbf{2}}\end{array}\right]^{\mathbf{\top}}$, substituting it into Eqn. (12) and simplifying the expression results in

$$
\left\|\mathrm{U}_{1}^{\mathrm{T}}\left(\mathrm{M}^{+}\right)_{\mathrm{vw}} \mathrm{e}_{\mathrm{y}}^{0}+\mathrm{S}_{1}\right\|^{2} \leq 1-\left\|\mathrm{U}_{2}^{\mathrm{T}}\left(\mathrm{M}^{+}\right)_{\mathrm{vw}} \mathrm{e}_{\mathrm{y}}^{0}\right\|^{2}
$$

This condition can only be satisfied when the right hand side is non-negative which further implies that

$$
\left|\mathrm{U}_{2}^{\top}\left(\mathrm{M}^{+}\right)_{\mathrm{vw}} \mathrm{e}_{\mathrm{y}}^{0}\right|^{2} \leq 1
$$

This condition and that imposed by Eqn. (6) are required for the existence of a validating model. If these conditions are not satisfied then the given nominal model and uncertainty structure cannot validate the input/output data. In such cases the nominal model and/or uncertainty structure and/or the definition of the external inputs must be modified.

Now define a variable $\phi$ such that

$$
\phi=\mathbf{U}_{\mathbf{1}}^{\mathbf{T}}\left(\mathbf{M}^{+}\right)_{v \mathbf{w}} \mathbf{e}_{\mathbf{y}}^{\mathbf{0}}+\mathbf{S}_{\mathbf{1}} \gamma .
$$

The condition in Eqn. (13) then becomes a constraint on $\phi$ such that

$$
\left\|\left.\phi\right|^{2} \leq 1-\right\| \boldsymbol{H}_{2}^{\mathrm{T}}\left(\mathrm{M}^{+}\right)_{\mathrm{vw}} \mathrm{e}_{\mathrm{y}}^{0} \|^{2} .
$$

Solving Eqn. (15) for $\gamma$ gives

$$
\gamma=\mathbf{S}_{\mathbf{1}}^{-\mathbf{1}}\left[\phi-\mathbf{U}_{\mathbf{1}}^{\mathbf{T}}\left(\mathbf{M}^{+}\right)_{\mathrm{vw}} \mathbf{e}_{\mathbf{y}}^{\mathbf{0}}\right] .
$$

There is an additional constraint on the selection of $\phi$ and $\psi$ associated with the structure of the uncertainty described in Eqn. (3). Assume that $\phi$ and $\psi$ satisfy the necessary conditions for existence of a 
validating model. Eqn. (7) can then be written in terms of $\phi$ and $\psi$ as follows.

$$
\begin{aligned}
\left\{\begin{array}{l}
\xi \\
\mathbf{v} \\
\mathbf{w}
\end{array}\right\}= & {\left[\mathbf{M}^{+}-\mathbf{N}_{\mathbf{M}} \mathbf{V}_{\mathbf{1}} \mathbf{S}_{\mathbf{1}}{ }^{-1} \mathbf{U}_{\mathbf{1}}^{\mathbf{T}}\left(\mathbf{M}^{+}\right)_{\mathbf{v w}}\right]_{\mathbf{y}}^{\mathbf{0}} } \\
& +\left[\begin{array}{ll}
\mathbf{N}_{\mathbf{M}} \mathbf{V}_{\mathbf{1}} \mathbf{S}_{\mathbf{1}}^{-\mathbf{1}} & \mathbf{N}_{\mathbf{M}} \mathbf{V}_{\mathbf{2}}
\end{array}\right]\left\{\begin{array}{l}
\phi \\
\psi
\end{array}\right\}
\end{aligned}
$$

Note, however, that the vector $\xi$ is not an external input, it depends directly on the uncertainty structure (see Figure 2) and needs to satisfy the following relations.

$$
\begin{gathered}
\xi=\Delta \mathbf{B} \Omega \eta \\
\left.\eta=\left[\begin{array}{ll}
\mathbf{P}_{\mathbf{1 1}} & \mathbf{P}_{\mathbf{1 3}}
\end{array}\right]\left\{\begin{array}{l}
\xi \\
\mathbf{v} \\
\mathbf{w}
\end{array}\right\}\right\}+\mathbf{P}_{\mathbf{1 2}} \mathbf{u}
\end{gathered}
$$

Substituting Eqn. (18) into Eqn. (20) results in the following equation.

$$
\begin{aligned}
& \eta=\left[\begin{array}{ll}
\mathbf{P}_{11} & \mathbf{P}_{\mathbf{1 3}}
\end{array}\right]\left[\mathbf{M}^{+}-\mathbf{N}_{\mathbf{M}} \mathbf{V}_{\mathbf{1}} \mathbf{S}_{\mathbf{1}}{ }^{-1} \mathbf{U}_{\mathbf{1}} \mathbf{T}\left(\mathbf{M}^{+}\right)_{\mathbf{v w}}\right] \mathbf{e}_{\mathbf{y}}^{\mathbf{0}} \\
& +\mathbf{P}_{12} \mathbf{u}+\left[\begin{array}{ll}
\mathbf{P}_{11} & \mathbf{P}_{13}
\end{array}\right]\left[\begin{array}{ll}
\mathbf{N}_{\mathbf{M}} \mathrm{V}_{\mathbf{1}} \mathbf{S}_{\mathbf{1}}{ }^{-1} & \mathbf{N}_{\mathbf{M}} \mathbf{V}_{\mathbf{2}}
\end{array}\right]\left\{\begin{array}{l}
\phi \\
\psi
\end{array}\right\}
\end{aligned}
$$

Therefore, given $\phi$ and $\psi$, the model validating solution is computed by Eqns. (18) and (21) subject to the further constraint that Eqn. (19) be satisfied.

Satisfying Eqn. (19) assures that the validating inputs determined by $\phi$ and $\psi, \mathbf{u}$, and $\mathbf{e}_{\mathbf{y}}^{\mathbf{0}}$ also generate $\eta$ and $\xi$ that satisfy the given uncertainty structure. Once such a solution is determined an actual uncertainty $\Delta_{\mathbf{B}}$ that achieves validation for the given input/output data and system model can be computed using Eqn. (19). Note that this computation is not required during the optimization used to compute $\phi$ and $\psi$. In addition, the solution to this equation is usually underspecified. That is, there are generally many possible choices for the elements of $\Delta_{\mathbf{B}}$ that satisfy the equation. A method for computing an optimal validating uncertainty will be presented subsequently.

In summary, the Lim-Giesy solution of the model validation problem boils down to (1) obtaining input/output data for the true system, (2) selecting a nominal linear model, a particular uncertainty structure, and set of external inputs, (3) testing whether the candidate model structure can generate a validating set of inputs and uncertainties, and (4) choosing $\phi$ and $\psi$ such that the model is validating, the external inputs are appropriately bounded, and the uncertainty structure is not violated. The following sections will build on this solution approach.

\section{Optimization Problem}

The largely arbitrary nature of $\phi$ and $\psi$ suggest that constrained optimization can be used to select them subject to the necessary constraints and additional user specified constraints and objectives. The necessary constraints are the bound on $\phi$ and the requirement that the uncertainty structure be satisfied. Additional constraints and objectives may be chosen to minimize the magnitude of some or all of the external inputs, minimize the magnitude of the uncertainties required to achieve validation, or trade off the magnitude of particular elements of the uncertainty structure.

The approach described in the literature ${ }^{[3]}$ is to place a scalar weighting factor $\rho$ on the uncertainty weighting matrix $\Omega=\rho \Omega_{0}$ (with $\Omega_{0}$ specified) that is minimized by selection of $\phi$ and $\psi$. In this way, the magnitude of the contribution of the uncertainty model to the validating response is minimized. This approach almost guarantees that the external inputs will take on values such that the norm bound condition in Eqn. (4) is active. This approach is fine if the magnitude of the external disturbances are known or if the uncertainty model is very simple (e.g., a single block). However, this is not usually the case.

A more comprehensive approach is to optimize over the weights on the uncertainty blocks $\omega_{\mathbf{i}}$ with the added objective of minimizing the magnitude of some or all of the elements of the external inputs $\mathbf{v}$ and $\mathbf{w}$. The cost function may be defined so as to seek the smallest possible norm of the uncertainty weight matrix $\Omega$ and of $\phi$ (which is essentially equivalent to minimizing the norm of the external inputs $\mathbf{v}$ and $\mathbf{w})$. This is the approach that has been implemented in the current research.

In more precise terms, assuming that none of the uncertainty blocks are associated with repeated scalar uncertainties, the problem statement is as follows. At each frequency for which input-output data is available

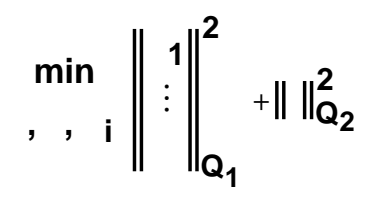

subject to the constraints

$$
\begin{array}{r}
\omega_{\mathbf{i}} \geq \frac{\left|\xi_{\mathbf{i}}\right|}{\left\|\eta_{i}\right\|}, \quad \mathbf{i}=12,3, \ldots, \tau \\
|\phi| \leq \sqrt{1-\mid \mathbf{U}_{2}^{\top}\left(\mathbf{M}^{+}\right)_{\mathbf{v w}} \mathbf{e}_{\mathbf{y}}^{0} \|^{2}}
\end{array}
$$


where $\mathbf{Q}_{\mathbf{1}}$ and $\mathbf{Q}_{\mathbf{2}}$ are diagonal semi-definite weighting matrices and $|(\cdot)|_{\mathbf{Q}}$ is the norm of the vector (.) with respect to the weighting matrix $\mathbf{Q}$.

At each frequency the objective function seeks to minimize, in a normed sense, the contributions of the various blocks of the uncertainty model while simultaneously minimizing, again in a normed sense, the size of the external inputs subject to two sets of constraints. The first constraint Eqn. (23a) insures that the validating inputs $\xi, \mathbf{v}$ and $\mathbf{w}$ are consistent with the structure of the uncertainty model determined by the $\omega_{\mathbf{i}}$ and $\Delta_{\mathbf{B}_{\mathbf{i}}}$ in Eqn. (3). That is, there exists an admissible uncertainty satisfying the norm bounds and block diagonal structure imposed by the uncertainty model. The second constraint Eqn. (23b) insures that the validating external inputs satisfy the bound condition from Eqn. (4).

If the uncertainty model further specifies that the structure of some of the uncertainty blocks represent repeated scalars such that

$$
\Delta_{\mathbf{B}_{\mathbf{i}}}=\delta \boldsymbol{\delta}_{\mathbf{i}} \mathbf{n}_{\mathbf{i}}
$$

where $\mathbf{I}_{\mathbf{i}}$ is the identity matrix of order $\mathbf{n}_{\mathbf{i}}$, then additional constraints must be imposed. The additional constraints are equality constraints and can be written

$$
\xi_{\mathbf{i}}=\omega_{\mathbf{i}} \delta_{\mathbf{i}} \eta_{\mathbf{i}}
$$

for those blocks that represent repeated scalar uncertainties where $\xi_{\mathbf{i}}$ and $\eta_{\mathbf{i}}$ are vectors corresponding to the ith uncertainty block $\Delta_{\mathbf{B}_{\mathbf{i}}}$. The scalar $\delta_{\mathbf{i}}$ can be real or complex valued depending on the uncertainty description but are always norm bounded by unity consistent with the definition of $\Delta$ B.

\section{Sequential Model Validation}

A further extension of the model validation approach is possible if elements of the uncertainty model is specified (i.e., one or more blocks of $\Delta_{\mathbf{B}}$ ). The effect of the specified elements on the model validating solution can be identified and eliminated from the solution of the remaining validating signals. This is done using an image space-null space decomposition similar to that used in the derivation of the model validation solution. ${ }^{[3]}$

Consider the situation in which the uncertainty structure is partitioned into two parts, one of which is assumed to be known a priori, $\Omega_{\mathbf{k}} \Delta_{\mathbf{B}_{\mathbf{k}}}$, and one that is to be determined, $\Omega_{\mathbf{u}} \Delta_{\mathbf{B}_{\mathbf{u}}}$. The constraint in Eqn. (19) can be written as two constraints on the elements of $\xi$ and $\eta$ associated with the two uncertainty model components, the first known and the second unknown.

$$
\begin{aligned}
& \xi_{\mathbf{k}}=\Delta_{\mathbf{B}_{\mathbf{k}}} \Omega_{\mathbf{k}} \eta_{\mathbf{k}}=\Delta_{\mathbf{k}} \eta_{\mathbf{k}} \\
& \xi_{\mathbf{u}}=\Delta_{\mathbf{B}_{\mathbf{u}}} \Omega_{\mathbf{u}} \eta_{\mathbf{u}}=\Delta_{\mathbf{u}} \eta_{\mathbf{u}}
\end{aligned}
$$

The fact that $\Omega_{\mathbf{k}} \Delta_{\mathbf{B}_{\mathbf{k}}}$ is specified influences the possible choices for $\phi$ and $\psi$ to achieve validation.

Consider the partitions of Eqns. (18) and (21) corresponding to the known uncertainty block. These can be written in terms of a linear equation in $\phi$ and $\psi$.

$$
\begin{aligned}
& \xi_{\mathbf{k}}=\xi_{\mathbf{k}}^{\mathbf{0}}+\mathbf{R}_{\mathbf{k}}\left\{\begin{array}{c}
\phi \\
\psi
\end{array}\right\} \\
& \eta_{\mathbf{k}}=\eta_{\mathbf{k}}^{\mathbf{0}}+\mathbf{T}_{\mathbf{k}}\left\{\begin{array}{l}
\phi \\
\psi
\end{array}\right\}
\end{aligned}
$$

where

$$
\begin{aligned}
& \xi_{\mathbf{k}}^{0}=\left[\left(\mathbf{M}^{+}\right)_{\mathbf{k}}-\left(\mathbf{N}_{\mathbf{M}}\right)_{\mathbf{k}} \mathbf{V}_{\mathbf{1}} \mathbf{S}_{\mathbf{1}}^{-1} \mathbf{U}_{\mathbf{1}}^{\mathbf{T}}\left(\mathbf{M}^{+}\right)_{\mathbf{v w}}\right] \mathbf{e}_{\mathbf{y}}^{\mathbf{0}} \\
& \eta_{\mathbf{k}}^{\mathbf{0}}=\left(\mathbf{P}_{\mathbf{1 2}}\right)_{\mathbf{k}} \mathbf{u}+\left[\begin{array}{ll}
\left(\mathbf{P}_{\mathbf{1 1}}\right)_{\mathbf{k}} & \left(\mathbf{P}_{\mathbf{1 3}}\right)_{\mathbf{k}}
\end{array}\right] \times \\
& {\left[\mathbf{M}^{+}-\mathrm{N}_{\mathbf{M}} \mathrm{V}_{\mathbf{1}} \mathrm{S}_{\mathbf{1}}{ }^{-1} \mathbf{U}_{\mathbf{1}}{ }^{\mathrm{T}}\left(\mathbf{M}^{+}\right)_{\mathrm{vw}}\right] \mathrm{e}_{\mathbf{y}}^{\mathbf{0}}}
\end{aligned}
$$

and where

$$
\begin{aligned}
& R_{k}=\left(N_{M}\right)_{k}\left[\begin{array}{ll}
V_{1} S_{1}{ }^{-1} & V_{2}
\end{array}\right] \\
& T_{k}=\left[\begin{array}{lll}
\left(P_{11}\right)_{k} & \left(P_{13}\right)_{k}
\end{array}\right] N_{M}\left[\begin{array}{ll}
V_{1} S_{1}^{-1} & V_{2}
\end{array}\right]
\end{aligned}
$$

The notation $(.)_{\mathbf{k}}$ represents the rows of (.) corresponding to the known uncertainty block and its inputs $\eta_{\mathbf{k}}$ and outputs $\boldsymbol{\xi}_{\mathbf{k}}$.

Substituting Eqn. (28) into the constraint in Eqn. (26) and solving for $\phi$ and $\psi$ results in the following expression.

$$
\left\{\begin{array}{l}
\phi \\
\psi
\end{array}\right\}=\mathbf{X}_{\mathbf{k}}^{+}\left(\xi_{\mathbf{k}}^{0}-\Delta \mathbf{k} \eta_{\mathbf{k}}^{0}\right)
$$

where

$$
\begin{gathered}
\mathbf{X}_{\mathbf{k}}=\Delta_{\mathbf{k}} \mathbf{T}_{\mathbf{k}}-\mathbf{R}_{\mathbf{k}} \\
\mathbf{X}_{\mathbf{k}}{ }^{+}= \begin{cases}\mathbf{X}_{\mathbf{k}}{ }^{\top}\left(\mathbf{X}_{\mathbf{k}} \mathbf{X}_{\mathbf{k}}{ }^{\top}\right)^{-1}, & \text { coldim }\left(\mathbf{X}_{\mathbf{k}}\right)>\operatorname{row} \operatorname{dim}\left(\mathbf{X}_{\mathbf{k}}\right) \\
\left(\mathbf{X}_{\mathbf{k}}{ }^{\top} \mathbf{X}_{\mathbf{k}}\right)^{-1} \mathbf{X}_{\mathbf{k}}{ }^{\top}, & \text { coldim }\left(\mathbf{X}_{\mathbf{k}}\right) \text { row } \operatorname{dim}\left(\mathbf{X}_{\mathbf{k}}\right)\end{cases}
\end{gathered}
$$

The right hand side of Eqn. (29) can be also written in terms of two components, one that lies in the image of $\mathbf{X}_{\mathbf{k}}$ and one that lies in the null space of $\mathbf{X}_{\mathbf{k}}$. The singular value decomposition of $\mathbf{X}_{\mathbf{k}}$ provides a means 
by which bases for these components can be determined.

$$
\mathbf{X}_{\mathrm{k}}=\left[\begin{array}{ll}
\mathbf{U}_{\mathrm{X}_{1}} & \mathrm{U}_{\mathrm{X}_{2}}
\end{array}\right]\left[\begin{array}{cc}
\mathrm{S}_{\mathbf{X}_{1}} & 0 \\
0 & 0
\end{array}\right]\left[\begin{array}{l}
\mathrm{V}_{\mathbf{X}_{1}}{ }^{\mathrm{T}} \\
\mathbf{V}_{\mathbf{X}_{2}}{ }^{\mathrm{T}}
\end{array}\right]
$$

The columns of the matrices $\mathbf{V}_{\mathbf{X}_{\mathbf{1}}}$ and $\mathbf{V}_{\mathbf{X}_{\mathbf{2}}}$ span the image and null spaces of $\mathbf{X}_{\mathbf{k}}$, respectively. Therefore, an expression for $\phi$ and $\psi$ can be written

$$
\left\{\begin{array}{l}
\phi \\
\psi
\end{array}\right\}=\mathbf{V}_{\mathbf{X}_{1}} \alpha+\mathbf{V}_{\mathbf{X}_{2}} \beta
$$

It is readily shown that substituting Eqn. (31) into Eqn. (29) simplifies to the following.

$$
\alpha=\mathbf{S}_{\mathbf{X}_{1}}{ }^{-1} \mathbf{U}_{\mathbf{X}_{1}}{ }^{\mathbf{T}}\left(\xi_{\mathbf{k}}^{0}-\Delta_{\mathbf{k}} \eta_{\mathbf{k}}^{0}\right)
$$

Note that the solution is independent of $\beta$. Also note that substituting Eqn. (32) into Eqn. (31) results in the following expression for $\phi$ and $\psi$ in terms of $\beta$.

$$
\left\{\begin{array}{l}
\phi \\
\psi
\end{array}\right\}=\mathbf{X}_{\mathbf{k}}^{+}\left(\xi_{\mathbf{k}}^{\mathbf{0}}-\Delta \mathbf{k} \eta_{\mathbf{k}}^{0}\right)+\mathbf{V}_{\mathbf{X}_{2}} \beta
$$

As a result, the constraint in Eqn. (26) is satisfied by Eqn. (33) and $\beta$ can be chosen to satisfy the remaining model validation relations and constraints. This is accomplished by substituting Eqn. (33) into the partitions of Eqns. (18) and (21) associated with the uncertainty block(s) remaining to be determined. The new model validation problem becomes finding a solution for $\beta$ that satisfies the combination of the following equations and the constraint in Eqn. (27).

$$
\begin{aligned}
& \left\{\begin{array}{c}
\xi_{u} \\
v \\
w
\end{array}\right\}=\left[\left(M^{+}\right)_{u v w}-\left(N_{M}\right)_{u v w} \mathbf{v}_{1} \mathbf{S}_{1}{ }^{-1} \mathbf{U}_{\mathbf{1}} \mathbf{T}\left(\mathbf{M}^{+}\right)_{\mathbf{v w}}\right] e_{\mathbf{y}}^{0} \\
& +\left(\mathbf{N}_{\mathbf{M}}\right)_{\mathrm{uvw}}\left[\begin{array}{ll}
\mathbf{V}_{\mathbf{1}} \mathbf{S}_{\mathbf{1}}^{-1} & \mathbf{V}_{\mathbf{2}}
\end{array}\right] \mathbf{X}_{\mathbf{k}}{ }^{+}\left(\xi_{\mathbf{k}}^{0}-\Delta_{\mathbf{k}} \eta_{\mathbf{k}}^{0}\right) \\
& +\left(\mathrm{N}_{\mathbf{M}}\right)_{\mathrm{uvw}}\left[\begin{array}{ll}
\mathrm{V}_{1} \mathrm{~S}_{1}^{-1} & \mathrm{~V}_{2}
\end{array}\right] \mathrm{v}_{\mathbf{X}_{2}} \beta \\
& \eta_{\mathbf{u}}=\left(\mathbf{P}_{\mathbf{1 2}}\right)_{\mathbf{u v w}} \mathbf{u} \\
& +\left[\begin{array}{ll}
\left(P_{11}\right)_{u v w} & \left(P_{13}\right)_{u v w}
\end{array}\right]\left[\mathbf{M}^{+}-\mathbf{N}_{\mathbf{M}} \mathbf{V}_{\mathbf{1}} \mathbf{S}_{\mathbf{1}}^{-1} \mathbf{U}_{\mathbf{1}}^{\mathbf{T}}\left(\mathbf{M}^{+}\right)_{\mathbf{v w}}\right] \mathbf{e}_{\mathbf{y}}^{\mathbf{0}} \\
& +\left[\begin{array}{ll}
\left(\mathbf{P}_{\mathbf{1 1}}\right)_{\mathrm{uvw}} & \left(\mathbf{P}_{\mathbf{1 3}}\right)_{\mathbf{u v w}}
\end{array} \mathbf{N}_{\mathbf{M}}\left[\begin{array}{ll}
\mathrm{V}_{\mathbf{1}} \mathbf{S}_{\mathbf{1}}^{-1} & \mathbf{V}_{\mathbf{2}}
\end{array}\right] \mathbf{X}_{\mathbf{k}}^{+}\left(\begin{array}{l}
\xi_{\mathbf{k}}^{0}-\Delta_{\mathbf{k}} \eta_{\mathbf{k}}^{\mathbf{0}}
\end{array}\right)\right. \\
& +\left[\begin{array}{ll}
\left(P_{11}\right)_{u v w} & \left(P_{13}\right)_{u v w}
\end{array}\right]_{M}\left[\begin{array}{ll}
V_{1} S_{1}^{-1} & V_{2}
\end{array}\right] V_{2} \beta
\end{aligned}
$$

Notice that the form of the expressions in Eqns. (34) and (35) is very similar to those in Eqns. (18) and (21). Each expression is the sum of a constant term and a term multiplying an unknown vector ( $\phi$ and $\psi$ in the earlier case and $\beta$ in this case). As a result, the same computing machinery used to solve the original model validation formulation can be used.

This new result provides a means for several variations of model validation that can be used in the solution of practical issues in uncertainty modeling. For example, the selection of the appropriate level of parameter uncertainty can be chosen by comparing (or optimizing) the level of additional uncertainty needed to achieve validation. This concept is developed further in the example section.

\section{Minimum Norm Validating Uncertainty}

The model validation based uncertainty modeling method provides uncertainty weights. It is useful to be able to compute an actual validating uncertainty (i.e., $\Omega$ and $\Delta_{\mathbf{B}}$ ) that has the same weights.

There are basically two types of validating uncertainty models to consider - one associated with repeated scalar blocks and one associated with full complex blocks. Both types result from the solution of the constraints in Eqn. (19) for a specific $\Omega \Delta_{\mathbf{B}}$ given an input-output pair and validating uncertainty weights. Recall that the validating uncertainty model is determined by solving for $\phi$ and $\psi$. The values of $\eta$ and $\xi$ can then be computed using Eqns. (18) and (20) and used to solve Eqn. (19).

Solving for a validating uncertainty associated with a repeated scalar block is trivial because the number of unknowns is the same as the number of equations, as is evident in Eqn. (25).

The solution of a validating uncertainty for a full complex block is more complicated because there are typically more unknowns than equations. One way to obtain a solution for a full complex block is via optimization. A family of validating uncertainties can be parameterized and these parameters can be optimized to satisfy Eqn. (19) and match the desired uncertainty weight.

Consider a set of candidate validating uncertainty blocks

$$
\Delta^{i}=\left[\begin{array}{lllll}
0 & \cdots & \delta^{i} & \cdots & 0
\end{array}\right], \quad i=1,2, \ldots, n
$$

where

$$
\delta^{\mathbf{i}}=\left\{\begin{array}{c}
\delta_{1 \mathbf{i}} \\
\vdots \\
\delta_{\mathrm{ni}}
\end{array}\right\}
$$

Substituting each block into Eqn. (19) simplifies to the following expression for the $\delta^{\mathbf{i}}$.

$$
\delta^{\mathbf{i}}=\xi / \eta_{\mathbf{i}}, \quad \mathbf{i}=1,2, \ldots, \mathbf{n}
$$


The sum of all the candidate solutions $\Delta^{\mathbf{i}}$ scaled by constant factors $\mathbf{k}_{\mathbf{i}}$ results in the following equation.

$$
\xi=\frac{\mathbf{1}}{\sum_{i=1}^{n} \mathbf{k}_{\mathbf{i}}}\left[\mathbf{k}_{\mathbf{1}}\left\{\delta^{\mathbf{1}}\right\} \quad \cdots \quad \mathbf{k}_{\mathbf{n}}\left\{\delta^{\mathbf{n}}\right\}\right] \eta
$$

Equation (39) describes a family of validating uncertainty blocks for arbitrary choices of the $\mathbf{k}_{\mathbf{i}}$.

A minimum norm validating uncertainty can be obtained by choosing the $\mathbf{k}_{\mathbf{i}}$ to minimize the difference between the specified uncertainty weight $\omega$ and the norm of the uncertainty matrix described in Eqn. (39), or equivalently

$$
\tilde{\mathbf{k}}_{\mathbf{i}}^{\min }|\omega-| \tilde{\Delta}|| \text { subject to } \sum_{\mathbf{i}=\mathbf{1}}^{\mathbf{n}} \tilde{\mathbf{k}}_{\mathbf{i}}=\mathbf{1}
$$

where

$$
\tilde{\Delta}=\sum_{\mathbf{i}=\mathbf{1}}^{\mathbf{n}} \tilde{\mathbf{k}}_{\mathbf{i}} \Delta^{\mathbf{i}}
$$

$\tilde{\Delta}$ is a minimum norm validating uncertainty.

\section{Examples}

Two examples will be presented to (1) demonstrate the result of optimizing the weighting for each block within a specified uncertainty structure, and (2) demonstrate the application of sequential model validation when part of the uncertainty model is known or otherwise specified.

\section{System Model $^{[3,5]}$}

Consider the simple physical system depicted in Figure 3. Two masses are connected to ground and each other via springs and dampers. The values of the masses, spring stiffness, and damping ratios are presented in Table 1. The inputs to this system are force actuators on the two masses and the outputs are the velocities of the two masses.

The equations of motion for the system in Figure 3 can be written

$$
\begin{gathered}
\left.\left.\left[\begin{array}{cc}
m_{1} & 0 \\
0 & m_{2}
\end{array}\right]\left\{\begin{array}{l}
\ddot{x}_{1} \\
\dot{x}_{2}
\end{array}\right\}+\left[\begin{array}{cc}
c_{1}+c_{2} & -c_{2} \\
-c_{2} & c_{2}+c_{3}
\end{array}\right]\right\} \begin{array}{l}
\dot{x}_{1} \\
\dot{x}_{2}
\end{array}\right\} \\
+\left[\begin{array}{cc}
k_{1}+k_{2} & -k_{2} \\
-k_{2} & k_{2}+k_{3}
\end{array}\right]\left\{\begin{array}{l}
x_{1} \\
x_{2}
\end{array}\right\}=\left\{\begin{array}{l}
f_{1} \\
f_{2}
\end{array}\right\} \\
y=\left[\begin{array}{ll}
1 & 0 \\
0 & 1
\end{array}\right]\left\{\begin{array}{l}
\dot{x}_{1} \\
\dot{x}_{2}
\end{array}\right\}
\end{gathered}
$$

These equations of motion can be written in modal coordinates and transformed into the frequency domain as a basis for demonstrating the model validation solutions described in the previous sections. The full

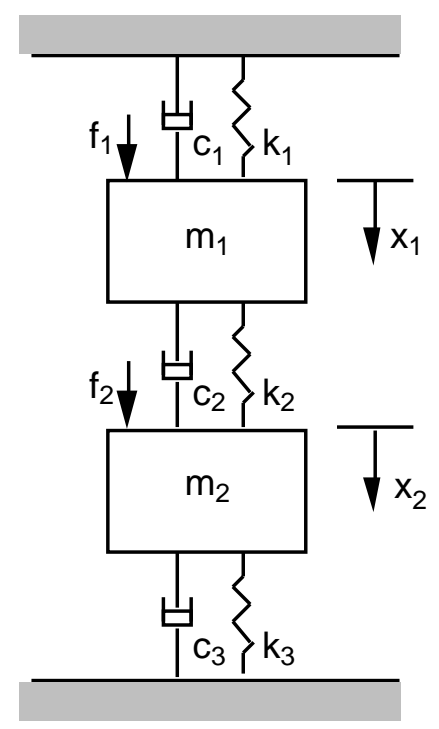

Figure 3 - Mass-spring-damper system.

Table 1 - Parameter values for mass-spring-damper system.

\begin{tabular}{c|r|r|r|r|r|r|r}
\multicolumn{2}{c|}{ Mass (kg) } & \multicolumn{3}{c|}{ Damping Ratio } & \multicolumn{3}{|c}{ Stiffness (N/m) } \\
\hline $\mathbf{m}_{\mathbf{1}}$ & $\mathbf{m}_{\mathbf{2}}$ & $\mathbf{c}_{\mathbf{1}}$ & $\mathbf{c}_{\mathbf{2}}$ & $\mathbf{c}_{\mathbf{3}}$ & $\mathbf{k}_{\mathbf{1}}$ & $\mathbf{k}_{\mathbf{2}}$ & $\mathbf{k}_{\mathbf{3}}$ \\
0.45 & 0.45 & 0.40 & 0.40 & 0.40 & 150 & 150 & 150
\end{tabular}

order system will be used as the "true system" shown in Figure 1. The nominal linear model is obtained by truncating the second (higher frequency) mode from the full order system. In this way the error between the true system and the nominal system is nothing more than the second mode of the full order system. In addition, the real and imaginary parts of the first mode eigenvalue are both decreased by 0.10 to represent parameter errors in the nominal model.

Model with Mixed Uncertainty

This example addresses the ability of the model validation approach to generate an uncertainty model associated with the combination of parameter errors and unmodeled dynamics in the mass-spring-damper system. Figure 4 depicts the uncertainty model structure in block diagram form. Two uncertainty blocks are considered - parameter uncertainty and additive uncertainty.

The additive uncertainty structure consists of (1) a full complex block $\Delta_{\mathbf{a}}$ in which each element of the $2 \times 2$ matrix can take on any complex value such that the norm of the matrix is less than unity, and (2) a 
single real scalar weight (i.e., $\Omega_{\mathbf{a}}=\omega_{\mathbf{a}} \mathbf{l}_{\mathbf{2}}$ ) that is chosen during the model validation solution.

The parameter uncertainty is modeled as repeated real scalar perturbations. The weighting and uncertainty matrices have the following structure.

$$
\begin{aligned}
& \Omega_{p}=\left[\begin{array}{rr}
\omega_{1}\left[\begin{array}{ll}
1 & 0 \\
0 & 1
\end{array}\right] & 0 \\
0 & \omega_{2}\left[\begin{array}{ll}
1 & 0 \\
0 & 1
\end{array}\right]
\end{array}\right] \\
& \Delta_{p}=\left[\begin{array}{rr}
\delta_{1}\left[\begin{array}{ll}
1 & 0 \\
0 & 1
\end{array}\right] & 0 \\
0 & \delta_{2}\left[\begin{array}{ll}
1 & 0 \\
0 & 1
\end{array}\right]
\end{array}\right]
\end{aligned}
$$

The first parameter $\delta_{1}$ is a perturbation of the real part of the nominal model eigenvalue and the second parameter $\delta_{\mathbf{2}}$ is a perturbation of the imaginary part. Each $\delta_{\mathbf{i}}$ is real valued and can have arbitrary values such that $\left|\Delta_{\mathbf{p}}\right| \leq 1$. Each $\omega_{\mathbf{i}}$ is also real but free to be chosen during the optimization.

The solution of the model validation problem is accomplished using the optimization described previously over a range of frequencies. The input vector is $\left[\begin{array}{ll}\mathbf{1} & -\mathbf{0 . 5}\end{array}\right]^{\boldsymbol{\top}}$ at each frequency. $\$$ The objective function is the sum squared value of the uncertainty weights $\omega_{\mathbf{i}}, \mathbf{i}=\mathbf{1}, \mathbf{2}, \ldots, \tau$. That is, the objective is to

$$
\min \left\|\begin{array}{c}
\omega_{1} \\
\vdots \\
\omega_{\tau}
\end{array}\right\|^{2} .
$$

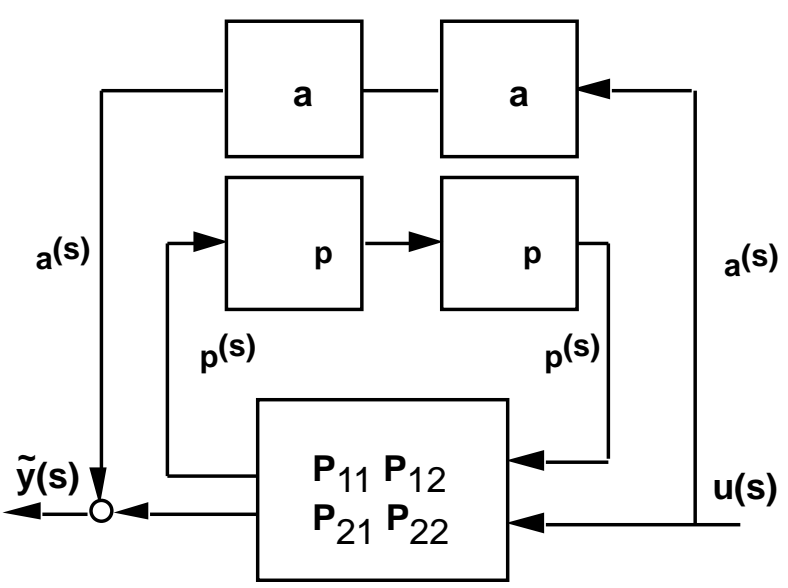

Figure 4 - Uncertainty model structure.

\footnotetext{
\#aplace transform of $\left[\begin{array}{c}1 \\ -0.5\end{array}\right] \delta(t)$.
}

For this example, the model error can be completely described by the chosen uncertainty structure. External disturbances and measurement noise are not considered and no weight is placed on the external disturbances.

Figure 5 depicts results from the model validation solutions. The three optimal uncertainty weights (i.e., the additive uncertainty weight and the two real parameter uncertainty weights) are plotted at each frequency at which a solution was obtained.

Figure 6 depicts the result of using the optimal uncertainty weights to generate a specific minimum norm validating uncertainty at each frequency point. The plot also shows several frequency responses of particular interest. The solid line is the frequency response of the "true system," the dashed line is the frequency response of the nominal model, and the dotted line is the frequency response associated with the error dynamics (i.e., the difference between the true system and the nominal model).

The circles are the result of solving for minimum norm validating uncertainties and using them to compute the frequency response of the uncertainty model that corresponds to the error dynamics of the system,

$$
\bar{\sigma}\left\{\mathbf{P}_{21}\left[\mathbf{I}-\tilde{\Delta} \mathbf{P}_{11}\right]^{-1} \tilde{\Delta} \mathbf{P}_{12}\right\} .
$$

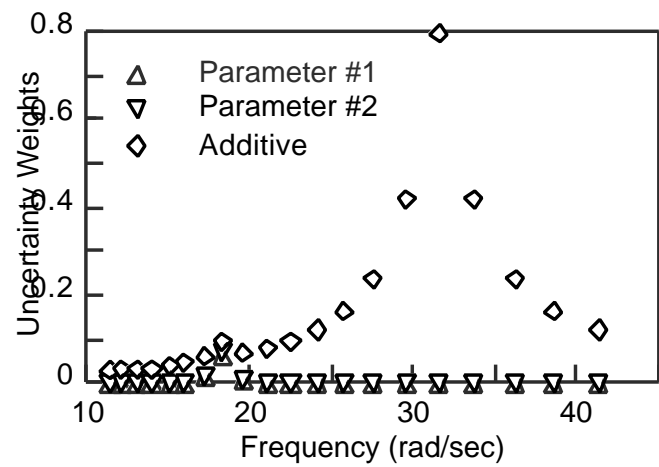

Figure 5 - Model validation uncertainty weights.

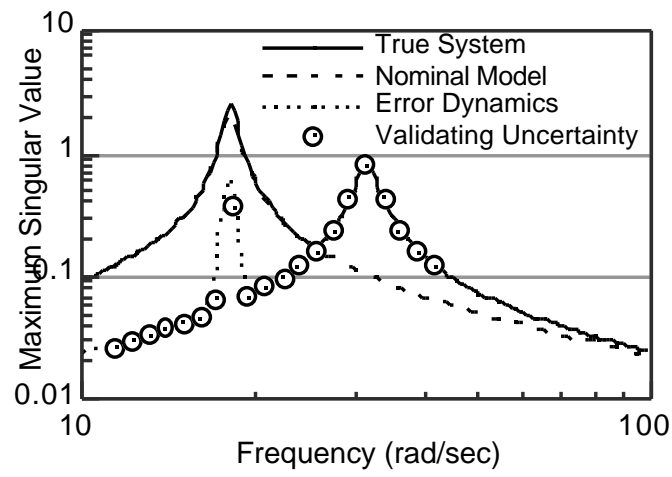

Figure 6 - Model validation solution. 
The minimum norm validating uncertainties provide a good approximation of the error dynamics. Therefore, the model validation based uncertainty weights accurately characterize the differences between the "true system" and the nominal model. However, one might also expect the real parameter uncertainty weights to be 0.1 at each frequency to reflect the actual parameter error. But this is not the case. So why are the real uncertainty weights so different from what might be expected?

Note that the contribution of the parameter error to the error dynamics is most significant in the frequencies near the first mode while the contribution of the unmodeled dynamics is most significant at frequencies near the second mode. At frequencies near the first mode (where the parameter uncertainty dominates) the weights associated with the real parameter uncertainty are close to the theoretically exact value of 0.1. At frequencies away from the first mode the weights associated with the real parameter uncertainty are very small.

The additive uncertainty is better able to characterize the uncertainty at frequencies away from the first mode than the parameter uncertainty. That is, the additive uncertainty permits validating signals for the system with a smaller weight than does the real parameter uncertainty. Recall that the solution approach seeks to find the smallest normed weights that achieve validation. The solution is not unique and a smaller norm solution than the theoretically exact solution was found to achieve model validation.

Also note that because the parameter error is independent of frequency it should be the same constant value at all frequencies. Therefore, one way to improve the uncertainty model would be to solve the model validation problem again and force the parameter uncertainty weights to take on the maximum value obtained during the mixed uncertainty solutions. The sequential validation approach is an effective way to solve this problem.

\section{Sequential Model Validation}

The following example parallels the previous example. However, in this case it is assumed that the parameter uncertainty is known a priori. That is, the parameter uncertainty matrix corresponds exactly to the difference between the parameters in the true system and the nominal model. Recall that for the massspring-damper system there were two uncertain parameters, the real and imaginary parts of the first mode of the system, both of which were decreased in the nominal model by 0.1 relative to their true values. be -

$$
\Omega_{p} \Delta_{p}=\left[\begin{array}{rrr}
0.1\left[\begin{array}{ll}
1 & 0 \\
0 & 1
\end{array}\right] & 0 \\
0 & 0.1\left[\begin{array}{ll}
1 & 0 \\
0 & 1
\end{array}\right]
\end{array}\right]
$$

The additive uncertainty weights were then computed from the optimization of $\beta$ to minimize the norm of the additive uncertainty weight and satisfy the constraint associated with its structure.

Figure 7 depicts results from the sequential validation solutions. The three uncertainty weights (i.e., the optimal additive uncertainty weight and the two specified real parameter uncertainty weights) are plotted as a function of frequency. Note that, in the frequency range near the first mode, the additive uncertain weight is smaller than the corresponding uncertainty weight from the mixed uncertainty result (see Figure 5). This is expected because the larger parameter uncertainty is now better able to characterize the errors.

The results of using the optimal uncertainty weights to compute a minimum norm validating uncertainties are shown in Figure 8. The plot shows the same frequency responses shown in Figure 6. However, the circles represent the uncertainty model dynamics associated with the optimized additive uncertainty weights with the real parameter weights specified. The

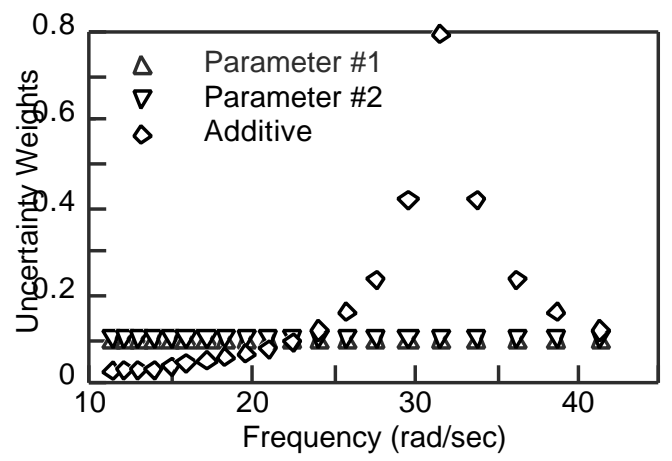

Figure 7 - Sequential validation uncertainty weights.

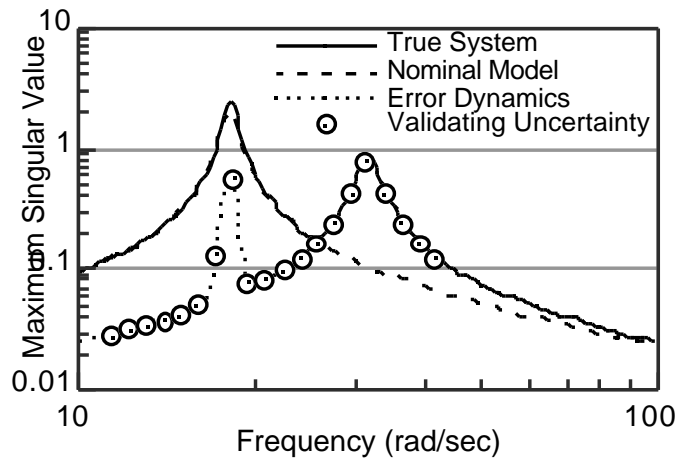

Figure 8 - Sequential validation frequency responses. 
minimum norm validating uncertainty provides a better approximation of the error dynamics than in the mixed uncertainty example (see Figure 6) as one might expect.

Had the specified value of the parameter uncertainty been in error, the solution of the sequential validation problem could still have been accomplished. However, the resulting additive uncertainty would have had to describe the additional uncertainty in the assumed model due to the remaining parameter errors. In fact, this can be used as a means for selecting the best parameter uncertainty if it is not known a priori.

The specified level of parameter uncertainty can be varied over a range of potential values. The sequential model validation method can then be used to compute the additional additive uncertainty required to achieve validation. A metric associated with the amount of additional uncertainty required to achieve validation can then be computed for each assumed level of parameter uncertainty. The parameter uncertainty producing the smallest additive uncertainty is the best choice for the mixed parameter / additive uncertainty structure.

The results from an example of this approach for the mixed uncertainty case is shown in Figure 9. The metric for additive uncertainty is the area under the plot of the additive uncertainty weight for a range of specified parameter weights. The smallest area occurs for parameter uncertainty of 0.1 which corresponds to the least amount of additional additive uncertainty required for validation and is equal to the actual parameter error introduced into the nominal model.

\section{Concluding Remarks}

The solution of the model validation problem developed by Lim and Giesy provides an excellent foundation for generating uncertainty models for robust control design applications. The extensions to the Lim-Giesy approach developed herein provide an ability to address some additional aspects of the uncertainty modeling problem. The extended optimization framework allows the determination of the best (in a normed sense) combination of uncertain elements (e.g., parameter and additive uncertainty) to achieve validation.

The sequential solution approach provides a means by which uncertainty structure can be addressed in the context of the standard model validation solution framework. This approach provides more control over the way in which various uncertain elements can be combined and does not require the engineer to rely solely on the optimizer to find the 'best' solution.

The sequential solution approach has some added benefit when there is more knowledge about the true system than just input/output data. For example, the

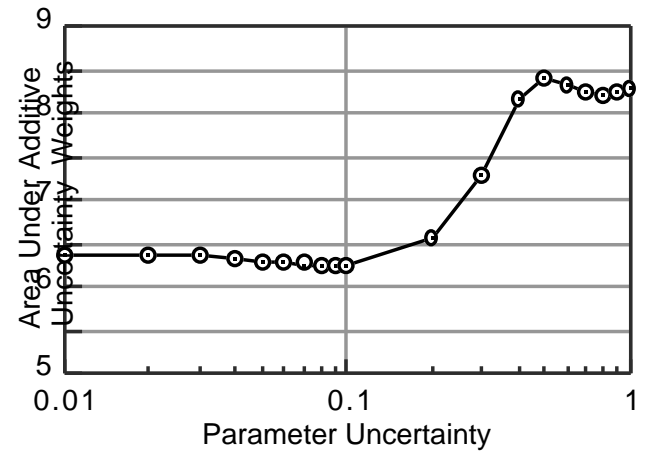

Figure 9 -Parameter uncertainty selection with sequential validation.

situation when certain parameter variations are known. This knowledge can be included in the model validation solution so that the additional uncertainty model components will only have to characterize those aspects of the system not otherwise described by the known components. The result is less conservative uncertainty models and thereby better controller designs.

\section{References}

[1] Maciejowski, J.M.: Multivariable Feedback Design. Addison-Wesley Publishers, Ltd. 1989.

[2] Balas, G.J.; Doyle, J.C.; Glover, K.; Packard, A.; and Smith, R.S.: $\mu$-Analysis and Synthesis Toolbox, User's Guide. The MathWorks, Inc. Natick, Mass. 1995.

[3] Lim, K.B. and Giesy, D.P.: Parameterization of Model Validating Sets for Uncertainty Bound Optimizations. AIAA Paper No. 98-4135.

[4] Smith, R.S. and Doyle, J.C.: Model Validation: A Connection Between Robust Control and Identification. IEEE Trans. on Automatic Control, Vol. 37, No. 7, July 1992. pp. 942952.

[5] Kumar, A. and Balas, G.J.: An Approach to Model Validation in the $\mu$ Framework. Proc. of the American Control Conference. Baltimore, Maryland. June 1994. pp. 3021-3026.

[6] Lim, K.B.; Cox, D.E.; Balas, G.J.; and Juang J.-N.: Validation of an Experimentally Derived Uncertainty Model. AIAA Paper No. 97-0244. 35th Aerospace Sciences Meeting and Exhibit. Reno, Nevada. January 6-10, 1997.

[7] Lim, K.B.; Balas, G.J.; and Anthony, T.C.: Minimum-norm Model Validating Identification for Robust Control. AIAA Paper No. 96-3717.

[8] Smith, R.S. and Doyle, J.C.: Towards a Methodology for Robust Parameter Identification. Proc. of the American Control Conference, 1990. pp. 2394-2399. 